\title{
Empleo de la fotogrametría de rango cercano y RPAs para la determinación de biomasa en parcelas agrícolas
}

\author{
José-Luis Marcos-Robles ${ }^{1}$, Luis Fernando Sánchez Sastre², María de la luz Gil Docampo ${ }^{3}$, \\ Juan Ortiz Sanz ${ }^{4}$, Santiago Martínez Rodríguez ${ }^{5}$
}

jlmarcos@iaf.uva.es, lux@iaf.uva.es,ml.gil@usc.es,juan.ortiz@es, azarelo@gmail.com

${ }^{1}$ Departamento de Ciencia de los Materiales, E.T.S.I.I.A.A., Universidad de Valladolid, Campus La Yutera Av Madrid 57, 34005, Palencia, España.

${ }^{2}$ Departamento de Ingeniería Agrícola y Forestal, E.T.S.II.AA., Universidad de Valladolid, Campus La Yutera Av Madrid 57, 34005, Palencia, España.

3,4,5 Departamento de Ingeniería Agroforestal, Universidad de Santiago de Compostela, EPS, Campus Universitario s/n, 27002 Lugo, España.

DOI: 10.17013/risti.19.118-130

Resumen: Para la estimación de carbono capturado por la biomasa aérea de un cultivo se suelen utilizar métodos destructivos basados en muestreos aleatorios de plantas. Dichos métodos requieren gran cantidad de tiempo, esfuerzo y coste económico, además del error generado en las interpolaciones. Por otra parte, la fotogrametría de rango cercano que aprovecha el uso de sensores aerotransportados en vehículos aéreos pilotados remotamente (RPAs o dron) pueden ser utilizados, de manera sencilla y rápida, para obtener la información necesaria en la estimación de la biomasa aérea de un cultivo, y por lo tanto del carbono asimilado. En este trabajo se propone una metodología basada en estas técnicas fotogramétricas utilizando cámaras ópticas convencionales aeroportadas en RPAs, con una velocidad de captura y procesamiento de datos alta, un relativo bajo coste y resultados más precisos y fiables.

Palabras-clave: carbono, dron, RPA, fotogrametría digital automatizada, MDT, MDS

\section{Use of close range photogrammetry and RPAs for determining biomass in agricultural areas}

Abstract: To estimate the carbon uptaken by the aerial biomass of a crop, destructive methods based on random sampling of plants are often used. These methods usually require a great effort of time, work and economic costs, besides the error generated in the interpolation process. On the other hand, close range photogrammetric methods that take advantage of sensors mounted on remotely piloted aircraft (RPAs or drone) can be used easily and quickly to obtain the necessary information in order to estimate the aerial biomass of a crop and therefore 
the assimilated carbon. In this work a methodology based on these photogrammetry techniques by using RPAs with optical off-the-shelf cameras onboard is proposed. This methodology allows a quick data acquisition and processing, relative low costs and reliable and accurate results.

Keywords: carbon, dron, RPA, Automated Digital Photogrammetry, DEM, DSM

\section{Introducción}

En el marco del proyecto LIFE11 ENV/ES/535 - Operation CO2 (http://operacionco2. com), cuyo objetivo principal es el secuestro de carbono mediante la conservación y manejo de bosques y las prácticas agroforestales integradas, se hacen estimaciones periódicas de biomasa en las labores de control y monitoreo, con el fin de evaluar la adicionalidad de carbono atribuible al proyecto. Una estimación precisa de biomasa es esencial para comprender mejor los ciclos de carbono en los ecosistemas terrestres, donde los bosques actúan como depósito primario del carbono (Houghton, 2005). Los métodos de campo tradicionales permiten evaluar la biomasa pero, por lo general, a un elevado coste ya que a menudo implica un muestreo destructivo en una cobertura geográfica limitada (Popescu, 2007).

Los métodos basados en sensores remotos, ya sean activos o pasivos, pueden realizar evaluaciones sobre el terreno para la estimación de la biomasa. Las ventajas de la utilización de estos sensores incluyen, entre otras, la posibilidad de obtener mediciones de cada lugar, la velocidad con la que los datos pueden ser recogidos y procesados, el relativamente bajo coste de muchos de estos sensores, y la capacidad de recopilar datos en áreas de difícil acceso desde el suelo (Bortolot \& Wynne, 2005; Sá et al., 2015).

A pesar de las ventajas del uso de estos sensores aeroportados, sigue siendo necesario capturar datos sobre el terreno que sirvan de apoyo y control. De esta forma, utilizando técnicas fotogramétricas y extrapolando los datos, se puede pasar de tener valores muestrales puntuales de una variable a tener una información continua de dicha variable en cualquier punto de la superficie de estudio (Marcos Robles et al., 2016).

Dentro de los sensores activos, la capacidad de los sensores LIDAR (Light Detection And Ranging) para medir directamente la estructura vegetal ha supuesto un gran avance en el inventario remoto de los recursos forestales (Sánchez Sastre et al., 2016). A pesar de que la tecnología LIDAR se postula como la más adecuada para la estimación de biomasa, actualmente el alto coste del hardware y de la ejecución de los vuelos, junto al elevado peso de los sensores, únicamente permite su aplicación en proyectos de gran envergadura o accediendo a los productos de planes públicos de ortofotografía (Porta et al., 2012). En proyectos más reducidos o con menor presupuesto, se plantean otros sistemas como los basados en sensores pasivos. Estos dependen de la variabilidad de las respuestas espectrales de la vegetación en las regiones del visible e infrarrojo cercano. Índices ampliamente aceptados como el NDVI (Índice de Vegetación de Diferencia Normalizada) y el LAI (Índice de Área Foliar) (Jonckheere, Nackaerts, Muys, van Aardt, \& Coppin, 2006; Morsdorf et al., 2004; Morsdorf, Kötz, Meier, Itten, \& Allgöwer, 2006) han sido empíricamente correlacionados con parámetros estructurales del dosel de copas. Pero mientras que los sistemas activos tipo LIDAR son capaces de ofrecer información precisa en ambas dimensiones, horizontal y vertical, el tratamiento digital 
de información multiespectral sólo proporcionan información en una de ellas. La teledetección, permite realizar mediciones detalladas de la distribución horizontal de la parte aérea de la planta (visible desde el aire) pero no permite extraer información de su estructura vertical con gran parte de sensores. Estudios basados en sensores ópticos (Marcos Robles et al., 2016) también proporcionan mediciones detalladas de la biomasa total. La fotogrametría de rango cercano permite calcular la biomasa aérea al permitir generar un Modelo de Elevación del Terreno (MDT).

Este trabajo tiene como objetivo estimar la biomasa en parcelas agrícolas utilizando sensores ópticos de espectro visible (RGB) elevados mediante plataformas áreas pilotadas remotamente a baja altitud (RPAs). El estudio se ha llevado a cabo en las diferentes zonas donde se desarrolla el proyecto.

\section{Material y métodos}

\section{1. Área de estudio}

El presente estudio, enmarcado dentro del proyecto LIFE+ Operation $\mathrm{CO} 2$, se ha realizado durante los meses de junio y julio de 2016 en tres parcelas sembradas con cultivos herbáceos anuales y localizadas en los siguientes municipios españoles: Soto de Cerrato (Palencia), Ayoó de Vidriales (Zamora) y San Mateo de Gállego (Zaragoza). Las superficies y los cultivos de trabajo fueron, respectivamente, 2,16 ha de alfalfa, 5,07 ha de triticale y 15,8 ha de centeno.

\subsubsection{Plataformas de vuelo}

Para realizar este ensayo se han utilizado tres plataformas de vuelo diferentes.

Mikrokopter® UFOCAMXXL8 V3: utilizado para volar en Soto de Cerrato. Es un vehículo aéreo de 8 hélices, autónomo, con despegue y aterrizaje vertical, que presenta un sistema de control simple y una elevada capacidad de carga de pago (ver Figura 1). Sus giros y desplazamientos se consiguen variando la potencia de uno o varios de los 8 rotores simultáneamente.

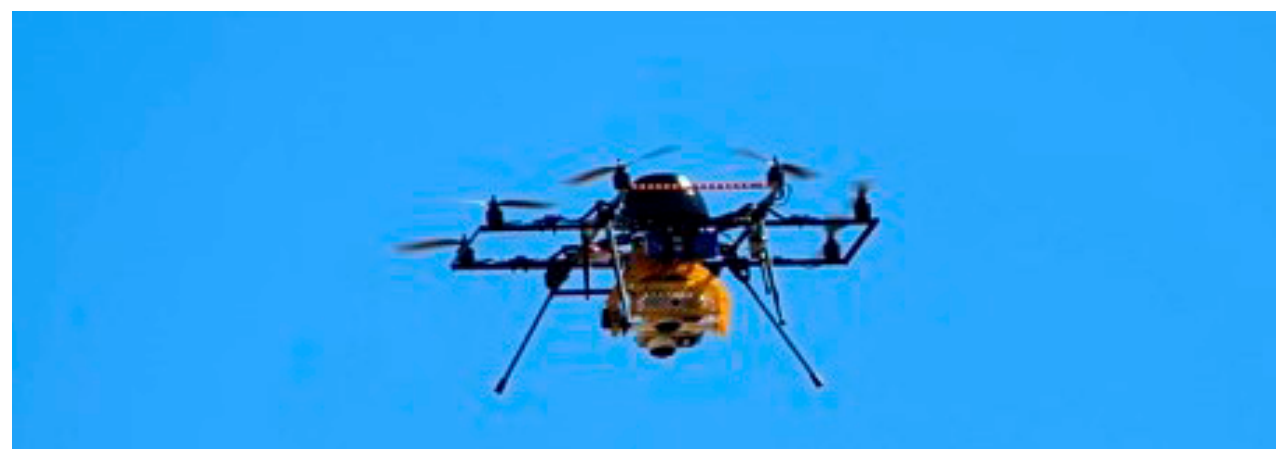

Figura 1 - Mikrokopter de 8 hélices con dos cámaras ejecutando un vuelo 
Está encuadrado en el grupo de las aeronaves de ala rotatoria y, por tener unas dimensiones pequeñas, en la subcategoría micro UAV (Nebiker, Christen, Eugster, Flückiger, \& Stierli, 2007). Es capaz de soportar un peso máximo de 2,5 kg, aunque a mayor peso menor rendimiento de la batería y por lo tanto menor autonomía de vuelo que, en condiciones normales, no supera los 12 minutos. Las condiciones meteorológicas exigidas para su buen funcionamiento son: humedad menor al 90\%, vientos máximos de $35-45 \mathrm{~km} / \mathrm{h}$ y temperaturas entre o y $40^{\circ} \mathrm{C}$. A esta aeronave se le ha adaptado un gimbal específico para poder integrar dos cámaras Olympus PEN E-P1, (una óptica y una modificada para captar el infrarrojo cercano) aunque para nuestra experiencia solamente emplea la óptica.

Skywalker kit: desarrollado por la empresa española Airelectronics y utilizado para volar en San Mateo de Gállego. Es un avión ligero de ala fija que se lanza a mano y que puede aterrizar prácticamente en cualquier superficie. El avión consta de un motor con una hélice, sistema GPS, sistemas de datos de vuelo, telemetría, control de cámaras añadiendo la georreferencia de las imágenes, emisora y aerofrenos que le pueden permitir acortar la maniobra de aterrizaje en caso de necesidad por fuertes vientos o zonas no demasiado visibles. Incorpora un autopiloto denominado U-Pilot $₫$, embebido en el fuselaje, que gobierna todos los sistemas de control de vuelo y de adquisición de imágenes. Su estructura está formada de materiales compuestos y poliolefina expandida (EPO) que hacen del avión un vehículo extremadamente ligero (1,3 kg sin contar con la carga de pago) y con una elevada autonomía de vuelo (superior a 40 minutos). Bajo las alas lleva adaptadas una cámara en el espectro visible modelo Canon A810 y una cámara multiespectral modelo Tetracam ADC Micro. El Skywalker no funciona aisladamente y para su correcto funcionamiento necesita, además, el enlace de comunicaciones y la estación de tierra. Alcanza una velocidad superior a los $50 \mathrm{~km} / \mathrm{h}$, pudiendo volar en condiciones adversas de vientos de hasta $40 \mathrm{~km} / \mathrm{h}$.

E-Bee $\AA$ : de la empresa Sensefly, perteneciente al grupo Parrot y utilizado para volar en Ayoó de Vidriales. Es una aeronave de ala fija, compacta, de $690 \mathrm{~g}$ de peso y $96 \mathrm{~cm}$ de envergadura construida con espuma de polipropileno expandido (EPP), estructura de carbono y partes de material compuesto. Posee un único motor sin escobillas de $160 \mathrm{~W}$ y tiene capacidad de transportar diferentes cargas de pago previamente adaptadas (cámaras fotográficas, multiespectrales, térmicas, etc.). La autonomía de vuelo alcanza los 50 minutos.

\subsection{Cámaras convencionales}

El Mikrokopter maneja una cámara fotográfica digital Olympus® Pen EPM-1 (12,3 Megapíxeles y sensor Live MOS de 4/3”). Se trata de una cámara cuyo sistema micro cuatro tercios posibilita el utilizar objetivos intercambiables, pero con un tamaño y peso menor (265 g el cuerpo con batería y tarjeta de memoria incluidas). Junto a la cámara se empleó el objetivo M.Zuikoß Digital ED 12 mm f/2 de 130 g de peso.

El Skywalker monta una cámara compacta, de la marca Canon $囚$, con un sensor CCD de 1/2.3 pulgadas de 16 Megapíxeles. Es muy ligera, apenas pesa 171 gr y tiene la posibilidad de poder adaptar un firmware modificado para facilitar las conexiones para el disparo remoto, que se conoce con el nombre de CHDK. Este programa permite la modificación 
de todos los parámetros importantes que se pueden registrar durante el vuelo, incluido la grabación en formato RAW.

El E-Bee utiliza una cámara digital compacta, la Sony® Cyber-Shot WX (18.2 Megapíxeles y sensor CCD tipo 1/2,3”). La aeronave se encarga de disparar automáticamente la cámara en función de la configuración de vuelo inicial.

\subsection{Software}

El procesado de las imágenes obtenidas con las cámaras fotográficas se realizó mediante el programa Agisoft PhotoScan® (PS) (Agisoft LLC, San Petersburgo, Rusia). Se trata de una de las principales soluciones informáticas de restitución fotogramétrica, de tipo comercial, disponibles actualmente que implementa la SFM-DMVR. Estas siglas hacen referencia a la Fotogrametría Digital Automatizada (SFM, Structure From Motion) y a los algoritmos de Reconstrucción en 3D de Alta Densidad a partir de Múltiples Puntos de Vista (DMVR, Digital Multi-View 3D Reconstruction), que permiten producir modelos 3D de alta precisión y calidad fotorrealista a partir de una colección de imágenes desordenadas de una escena u objeto, tomadas desde diferentes puntos de vista. Se trata de conseguir una sola imagen que contenga la información requerida a partir de la fusión de múltiples imágenes capturadas mediante la plataforma pertinente (Medina et al., 2013). La gran precisión del sistema (Doneus et al., 2011) y la baja desviación existente frente a los modelos obtenidos a partir de datos provenientes de escáneres láser son avaladas en múltiples trabajos (Rodriguez-Navarro, 2012).

Como en todo análisis de información geospacial se hace necesario el uso de herramientas SIG (Sistemas de Información Geográfica) capaces de integrar datos geoespaciales y descriptivos (Tolaba et al., 2014). En este caso, el procesado de los modelos digitales del terreno se realiza a través de Global Mapper@ (Blue Marble Geographics, Maine, Estados Unidos) que es un software de análisis y procesamiento de datos SIG. El programa puede ejecutarse de forma autónoma o bien integrado como un módulo en otras herramientas SIG, y tiene capacidad para acceder a gran cantidad de repositorios de imágenes, datos topográficos y archivos MDT / MDS. Permite cálculos de distancias y superficies, análisis espectral, cálculo de volúmenes de corte y relleno, etc., además, es también compatible con datos LIDAR.

\section{Metodología}

La altura de vuelo óptima se estableció entre 75-100 metros (2-3 cm de resolución espacial, dependiendo de la cámara utilizada) y el solape mínimo, longitudinal y transversal en 70\% y 40\% respectivamente, para garantizar la correcta generación del modelo 3D con Agisoft PhotoScan $囚$.

El número de puntos de control (PC), variable en función del tamaño de la parcela, debe ser mayor o igual a 10. Estos han de repartirse sobre el terreno, cubriendo la zona central y los extremos de la parcela, y ser geolocalizados con GPS de precisión centimétrica (ver Figura 2). Los puntos deben ser definidos sobre elementos altamente identificables en las fotografías tomadas desde el RPA (marcas predefinidas o dianas) y nos van a servir en la elaboración de los MDS. 


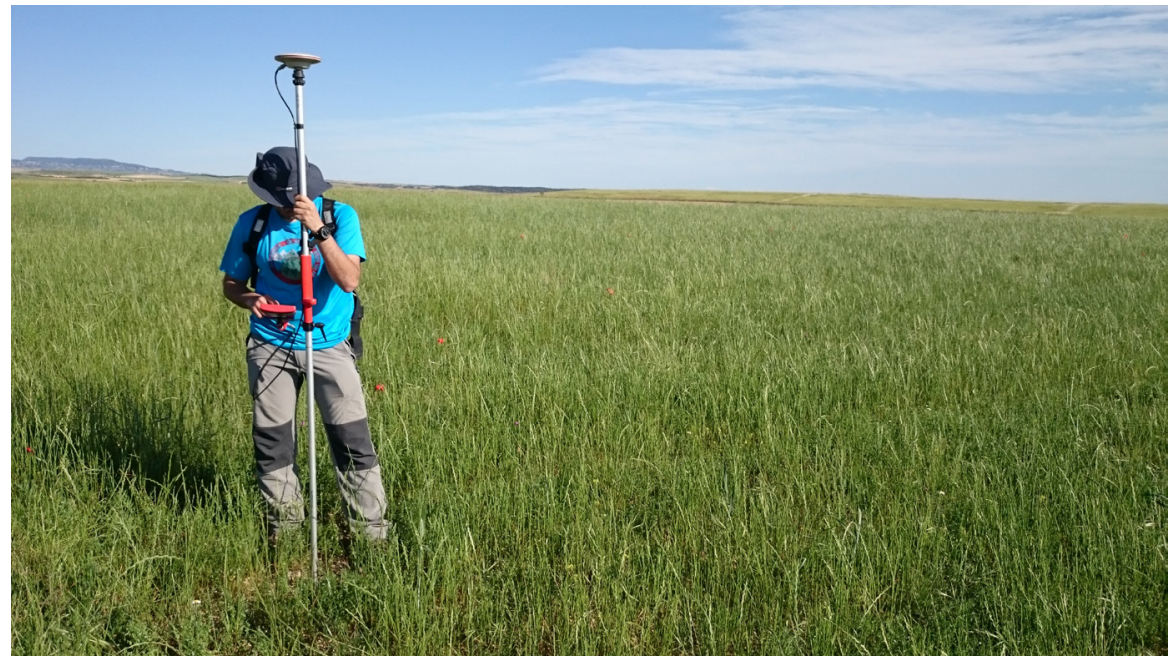

Figura 2 - Toma de punto de control con GPS de precisión centimétrica

Tras la finalización del vuelo se procede a realizar el cálculo de biomasa por muestreo aleatorio de un mínimo de 3 puntos. Se trata de un muestreo de carácter destructivo y por ello debe realizarse a posteriori. En cada punto se lanza un aro de superficie conocida $\left(0,25 \mathrm{~m}^{2}\right)$, se georreferencia y se extraen las plantas que se encuentren en su interior (parte aérea).

Posteriormente, en el laboratorio, las plantas de cada muestra se pesan en húmedo, se secan y después se vuelven a pesar en seco. Los valores en peso que se obtienen, se extrapolarán posteriormente a toda la superficie de la parcela.

\subsection{Corrección de las coordenadas GPS}

Las coordenadas GPS de todos PC se corrigen aumentando en 1 metro su cota para evitar la aparición de zonas con volumen negativo entre el modelo digital de superficie (MDS) generado con PhotoScan y el modelo digital del terreno (MDT) obtenido a través del Plan Nacional de Ortofotografía Aérea (PNOA) del Instituto Geográfico Nacional. Este error proviene fundamentalmente de obtener mayor resolución espacial del MDS generado con PhotoScan respecto al MDT del PNOA. Al aumentar la cota de los PC conseguimos que el MDS tenga una mayor cota también, consiguiendo así que la diferencia entre ambos modelos digitales sea positiva en toda la superficie.

Esto supone más volumen por unidad de superficie, sin embargo, no afecta a los cálculos de biomasa, ya que el Factor de Densidad $\left(\mathrm{Kg} / \mathrm{m}^{3}\right)$ que calculamos posteriormente se encarga de corregirlo (al aumentar el volumen, el factor de densidad decrece).

\subsection{Creación del modelo digital de superficie}

A partir de las fotografías capturadas con el RPA (ver Figura 3) y los PC, se genera un modelo $3 \mathrm{D}$ de la superficie con la mayor densidad de malla posible (entre calidad "Media" y "Ultra Alta", dependiendo del número de fotos y las capacidades del equipo 
informático). Es importante limitar la densidad de caras de la malla a 10 millones para evitar que se colapse el ordenador.

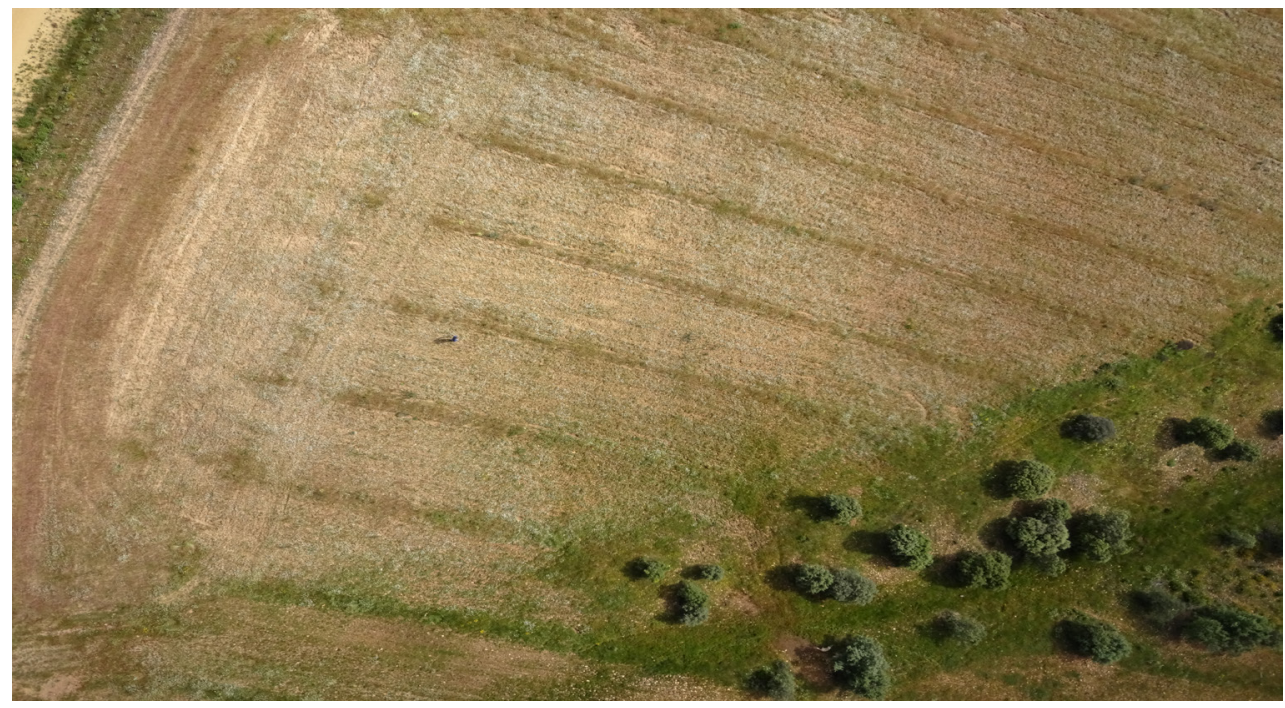

Figura 3 - Ejemplo de fotografía tomada con en Ayoó de Vidriales

Tras ello, se exporta el MDS de cada parcela al formato TIF (GeoTIFF Elevation Data). Este formato integra la información geográfica asignada al modelo 3D por medio de los PC. Paralelamente, también a partir del conjunto de fotografías obtenidas desde el RPA se puede obtener una ortofoto a modo de mosaico (ver Figura 4).

\subsection{Cálculo del volumen por unidad de superficie de la parcela y de los puntos de muestreo de biomasa}

Se cargan los modelos digitales de elevación (MDT y MDS) en el programa Global Mapper y se generan los recortes de las zonas de interés, de las cuales queremos obtener el volumen y su correspondiente superficie. Estas son:

- Toda la superficie objeto de la medición de biomasa. Se eliminan las calles u otra superficie que no se corresponda con la zona de trabajo. La idea es obtener el MDS únicamente de la zona de vegetación cuya superficie se corresponda con la superficie final en la cual se va a estimar la biomasa.

- Cada una de las zonas anexas a los puntos de muestreo en los que se tomaron datos de biomasa en campo.

Con ello definimos el volumen de vegetación por unidad de superficie de cada parcela y de los puntos de muestreo de biomasa, en este caso en $\mathrm{m}^{3} / \mathrm{ha}$.

\subsection{Cálculo del factor de densidad}

Para poder estimar la biomasa de cada una de las parcelas es necesario calcular un factor de densidad que nos permita pasar de $\mathrm{m}^{3} / \mathrm{ha}$ a $\mathrm{kg} / \mathrm{ha}$. Esto se consigue gracias a los 

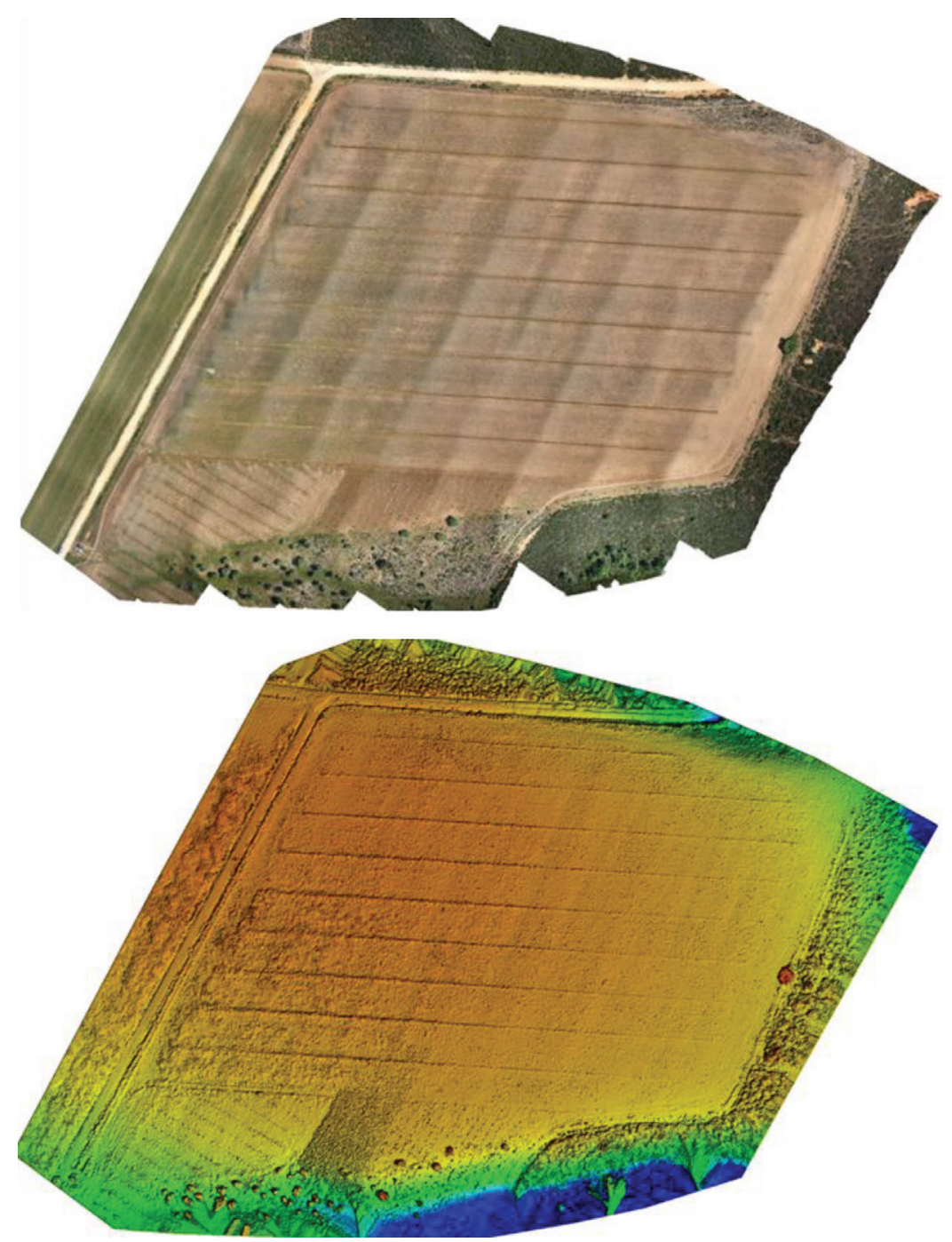

Figura 4 - Ortofoto de la parcela de Ayoó de Vidriales obtenida a partir de las fotos tomadas durante el vuelo (arriba) y MDS de la misma superficie (abajo)

puntos de muestreo de biomasa medidos en campo. Con el dato de volumen y peso por unidad de superficie de cada punto de muestreo, se aplica la siguiente fórmula para calcular su factor de densidad:

$$
F d=\frac{B}{V}
$$


Donde:

- $\quad F d$ : factor de densidad $\left(\mathrm{kg} / \mathrm{m}^{3}\right)$,

- B: biomasa por unidad de superficie (kg/ha) y

- $\quad \mathrm{V}$ : Volumen por unidad de superficie $\left(\mathrm{m}^{3} / \mathrm{ha}\right)$.

Cabe reseñar que el factor de densidad no representa ninguna característica física de la parcela, sino la relación entre el volumen que detecta PhotoScan y los kilogramos medidos en cada punto mediante los muestreos realizados en campo. PhotoScan calcula un volumen mayor si la capa vegetal es densa y tupida y menor en caso contrario. Este factor nos permite corregir estas variaciones y calcular de forma objetiva la biomasa en el resto de la parcela.

\subsection{Cálculo de biomasa por unidad de superficie y biomasa total (biomasa aérea)}

El primer paso es definir el factor de densidad de cada parcela como la media de los factores de densidad de los puntos de muestreo. Con el valor obtenido y aplicando la siguiente fórmula, se obtiene el valor de biomasa por unidad de superficie:

$$
B=F d \times V
$$

Donde:

- B: biomasa por unidad de superficie ( $\mathrm{kg} / \mathrm{ha})$,

- Fd: factor de densidad de la parcela $\left(\mathrm{kg} / \mathrm{m}^{3}\right) \mathrm{y}$

- V: Volumen por unidad de superficie $\left(\mathrm{m}^{3} / \mathrm{ha}\right)$.

- BT: biomasa total de la parcela $(\mathrm{kg})$,

- B: biomasa por unidad de superficie $(\mathrm{kg} / \mathrm{ha})$ y

- S: Superficie real de la parcela (ha).

\section{Resultados}

\subsection{Datos de campo}

En San Mateo de Gállego, se han tomado 10 PC distribuidos por toda la parcela. Para la toma de fotografías con el Skywalker, se realizó un único vuelo en el que se tomaron 149 imágenes de la zona de trabajo a una altura sobre el terreno de 214 metros. El tamaño medio del píxel sobre el terreno es de $5 \mathrm{~cm} /$ píxel.

En Ayoó de Vidriales se han tomado 11 PC distribuidos por toda la parcela. Para la toma de fotografías con el E-Bee se realizó un único vuelo en el que se tomaron 427 imágenes de la zona de trabajo a una altura sobre el terreno de 84,7 metros. A menor altura sobre el terreno, mayor resolución. El tamaño medio del píxel sobre el terreno es de $2,15 \mathrm{~cm} /$ píxel. 
En Soto de Cerrato se han empleado 5 PC distribuidos uniformemente por toda la parcela. Para la toma de fotografías con el Mikrokopter se realizó un único vuelo en el que se tomaron 136 imágenes de la zona de trabajo a una altura sobre el terreno de 77,4 metros. En este caso, por volar a una baja altura y portar una cámara de excelente resolución, se pudo alcanzar un tamaño medio del píxel sobre el terreno de 1,9 cm/píxel.

A continuación, se presenta la tabla 1 en la que se relaciona los puntos de muestreo medidos en campo con cada una de las parcelas.

\begin{tabular}{lll}
\hline Parcela & Puntos de muestreo & $\begin{array}{l}\text { Biomasa }(\text { (kg/ha) Producción } \\
\text { grano }\end{array}$ \\
\hline 1 & Punto 1 & 2.868 \\
\hline 1 & Punto 2 & 3.150 \\
\hline 2 & Punto 3 & 2.740 \\
\hline 2 & Punto 1 & 1.700 \\
\hline 2 & Punto 2 & 1.560 \\
\hline 3 & Punto 3 & 1.920 \\
\hline 3 & Punto 1 & 3.289 \\
\hline 3 & Punto 2 & 3.321 \\
\hline
\end{tabular}

Tabla 1 - Puntos de muestreo de biomasa medidos en campo (Parcela 1: San Mateo de Gállego;

Parcela 2: Ayoó de Vidriales; Parcela 3: Soto de Cerrato)

\subsection{Datos de gabinete}

Seguidamente se muestra la tabla 2 con todos los resultados extraídos del trabajo de gabinete gracias a la aplicación PhotoScan (creación del MDS) y Global Mapper (medición del volumen entre el MDS de la parcela y el MDT) y la tabla 3 con el Factor de Densidad calculado de cada parcela y los kg totales de biomasa obtenidos en cada una de ellas.

\begin{tabular}{llll}
\hline Parcela & $\begin{array}{l}\text { Sup. Real } \\
\left(\mathbf{m}^{\mathbf{2}}\right)\end{array}$ & $\begin{array}{l}\text { Volumen total }(\mathbf{m} 3) \\
(\mathbf{M D T}-\mathbf{M D S z})\end{array}$ & $\begin{array}{l}\text { Volumen } / \text { ud superf. } \\
(\mathbf{m} \mathbf{3} / \mathbf{h a})\end{array}$ \\
\hline 1 & 50.700 & 70.633 & 13.932 \\
\hline 2 & 158.000 & $171.914,1$ & 10.881 \\
\hline 3 & 21.650 & $17.243,7$ & 7.965 \\
\hline
\end{tabular}

Tabla 2 - Resultados extraídos del MDSz de la parcela

\begin{tabular}{llll}
\hline Parcela & Fd $\left(\mathbf{k g} / \mathbf{m}^{3}\right)$ & Biomasa (kg/ha) & Biomasa total $(\mathbf{k g})$ \\
\hline 1 & 0,213 & 2.971 (grano) & 15.065 (grano) \\
\hline 2 & 0,158 & 1.714 (grano) & 27.088 (grano) \\
\hline 3 & 0,367 & 2.923 (forraje) & 6.329 (forraje) \\
\hline
\end{tabular}

Tabla 3 - Resultado de la biomasa obtenida en las 3 parcelas. Fd corresponde al factor de densidad 


\section{Conclusiones}

El estudio ha permitido determinar la biomasa de las tres parcelas sembradas mediante el uso de la metodología propuesta, obteniendo resultados que guardan una gran coherencia con lo observado en campo. Esto indica que los protocolos de recolección de datos de campo y planificación de vuelos, así como la metodología de post-proceso han sido fiables y consistentes.

En el mismo sentido, la toma de datos de alta precisión utilizada en la metodología ha permitido obtener resultados de calidad del parámetro biomasa, tanto en producción de grano como de biomasa total. No obstante, se ha visto necesario adoptar precauciones durante todo el proceso como, por ejemplo, que la toma de los PC y los puntos de muestreo de biomasa en campo se haga con apoyo de GPS de alta precisión (con errores por debajo de los $3 \mathrm{~cm}$ ), o que los vuelos se planifiquen correctamente manteniendo un área de seguridad para evitar los problemas de pérdida de información en los bordes de la parcela.

La utilización de multirrotores de escasa autonomía de vuelo (como en el caso de la parcela de Soto de Cerrato), obliga a la creación de subparcelas de muestreo en parcelas de gran tamaño. Esto implica la introducción de un error por extrapolación de los resultados parciales a toda la superficie y una pérdida de objetividad en el cálculo de la biomasa total. Sin embargo, gracias a la utilización de RPAs de ala fija en las parcelas de mayor tamaño (San Mateo de Gállego y Ayoó de Vidriales) se ha logrado medir toda la superficie en un único vuelo reduciendo los costes en el trabajo de campo (tiempo de vuelo) y de gabinete (aunque es mayor el volumen de fotografías, únicamente se genera un modelo 3D). El mayor problema que pueden presentar los RPAs de ala fija es la menor nitidez de las imágenes debido a la menor estabilidad de este tipo de aeronaves y a la mayor velocidad que alcanzan frente a las de ala rotatoria. No obstante, se ha comprobado que las imágenes tomadas con el E-Bee son de mayor calidad que las del Skywalker. Esto puede deberse a la mayor la calidad de su sensor y a la menor altura de vuelo.

Por otra parte, al haberse empleado tres plataformas de vuelo distintas con cámaras de diferentes características, podría ser interesante analizar, en estudios futuros, cómo afecta el binomio cámara/RPA a la calidad de los resultados. Para ello, sería necesario realizar varias repeticiones de los vuelos (entre 5 y 10) con cada dron y tomar un mayor número de muestras de biomasa en campo (unas 20-30 con su correspondiente ubicación GPS de precisión centimétrica).

En resumen, el empleo de RPAs junto con la fotogrametría digital automatizada en este proyecto, han permitido estimar la cantidad de biomasa existente en la totalidad de la superficie de las parcelas estudiadas, a partir de la información obtenida de los sensores ópticos aeroportados mediante drones. De este modo, se reducen los errores por interpolación de datos muestrales o por poca representatividad de los mismos. Además, se reducen significativamente los esfuerzos de tiempo, trabajo sobre el terreno y costes a largo plazo. Por lo tanto, estas tecnologías constituyen un método apto para la determinación de biomasa aérea de cultivos y, por lo tanto, de la cantidad de carbono retenido en los mismos. 


\section{Agradecimientos}

Los autores agradecen al proyecto europeo del Programa LIFE+ "Operación CO2", así como a los Ayuntamientos de San Mateo de Gállego (Zaragoza) y Ayoó de Vidriales (Zamora) y al personal de mantenimiento de los campos experimentales de la UVa en Soto de Cerrato (Palencia).

\section{Referencias bibliográficas}

Bortolot, Z. J., \& Wynne, R. H. (2005). Estimating forest biomass using small footprint LiDAR data: An individual tree-based approach that incorporates training data. ISPRS Journal of Photogrammetry and Remote Sensing, 59(6), 342-360.

Doneus, M., Verhoeven, G., Fera, M., Briese, C., Kucera, M., \& Neubauer, W. (2011). From deposit to point cloud-a study of low-cost computer vision approaches for the straightforward documentation of archaeological excavations. Geoinformatics FCE CTU, 6, 81-88.

Houghton, R. A. (2005). Aboveground forest biomass and the global carbon balance. Global Change Biology, 11(6), 945-958.

Jonckheere, I., Nackaerts, K., Muys, B., van Aardt, J., \& Coppin, P. (2006). A fractal dimension-based modelling approach for studying the effect of leaf distribution on LAI retrieval in forest canopies. ecological modelling, 197(1), 179-195.

Marcos Robles, J.L., Gil Docampo, M.L., Ortiz Sanz, J., Martínez Rodríguez, S., Garrido Lagunaga, F., Sánchez Sastre, L.F., ... Ortiz Sanz, L. (2016). Determinación de biomasa en parcelas de cultivos herbáceos mediante cámaras ópticas elevadas por medio de vehículos aéreos no tripulados (UAV). In Proceedings of the 6th IberoAmerican Congress on Qualitative Research (CIAIQ2016), Qualitative Research on Engineering and Technology, vol 4. Porto, Portugal.

Medina, J., Joyanes., L. \& Pinilla, C. (2013). Aplicativo Web para la Fusión de Imágenes Satelitales. RISTI - Revista Ibérica de Sistemas e Tecnologias de Informação, (11), 17-30. doi: 10.4304/risti.11.17-30

Morsdorf, F., Kötz, B., Meier, E., Itten, K. I., \& Allgöwer, B. (2006). Estimation of LAI and fractional cover from small footprint airborne laser scanning data based on gap fraction. Remote Sensing of Environment, 104(1), 50-61.

Morsdorf, F., Meier, E., Kötz, B., Itten, K. I., Dobbertin, M., \& Allgöwer, B. (2004). LIDAR-based geometric reconstruction of boreal type forest stands at single tree level for forest and wildland fire management. Remote Sensing of Environment, 92(3), 353-362.

Nebiker, S., Christen, M., Eugster, H., Flückiger, K., \& Stierli, C. (2007). Integrating mobile geo sensors into collaborative virtual globes-design and implementation issues. En Mobile Mapping Technologies Symposium MMT. Citeseer. 
Popescu, S. C. (2007). Estimating biomass of individual pine trees using airborne lidar. Biomass and Bioenergy, 31(9), 646-655.

Porta, J., Parapar, J., García, P., Fernández, G., Touriño, J., Ónega, F., ... Crecente, R. (2012). Sistema de Información del Banco de Tierras de Galicia. RISTI -Revista Ibérica de Sistemas e Tecnologias de Informação, 9, 27-41. doi: 10.4304/ risti.9.27-41.

Rodriguez-Navarro, P. (2012). Automated Digital Photogrammetry versus the systems based on active $3 \mathrm{D}$ sensors. La Fotogrametría Digital Automatizada frente a los sistemas basados en sensores 3D activos. EGA, 17(20), 100-111.

Sá, F., Rocha, Á., \& Cota, M. P. (2015). Preliminary dimensions for a quality model of Electronic Local Government services. In 2015 1oth Iberian Conference on Information Systems and Technologies (CISTI) (pp. 1-10). IEEE.

Sánchez Sastre, LF., Herrero Llorente, E., Hernández Navarro, S., Carrión Prieto, P., Garrido Lagunaga, F., Marcos Robles, J.L. \& Clérigo Pérez, Z. (2016). Proyecto LIFE+ Operación CO2: nuevas tecnologías aplicadas a la obtención de créditos de carbono mediante reforestación, restauración y gestión forestal. In Proceedings of the 6th Ibero-American Congress on Qualitative Research (CIAIQ2016), Qualitative Research on Engineering and Technology, vol 4. Porto, Portugal.

Tolaba, A.C., Caliusco, M.L. \& Galli, M.R. (2014). Representación del Conocimiento de la Información Geográfica siguiendo un Enfoque basado en Ontologías. RISTI Revista Ibérica de Sistemas e Tecnologias de Informação, (14), 101-116. doi: 10.17013/risti.14.101-116 